Petr Hruby - Tomas Nahlik - Dana Smetanova*

\title{
MATHEMATICAL MODELLING OF SHAFTS IN DRIVES
}

Propeller shafts of the vehicle's drive transmit a torque to relatively large distances. The shafts are basically long and slender and must be dimensioned not only in terms of torsional stress, but it is also necessary to monitor their resistance to lateral vibration.

In the paper, a simple model (of the solved problem) is constructed by the method of physical discretization, which is evident from the nature of the centrifugal force fields' influence on the spectral properties of the shaft. An analytical solving of speed resonances prop shafts test model (whose aim is to obtain values for verification subsequently processed models based on the transfer-matrix method and the finite element method) is performed.

Keywords: Hook's joint, shaft, vibration, mathematical and physical model, transfer matrix, Finite element method

\section{Introduction}

Problem of modelling and description of propeller shafts is quite old [1]. Propeller shafts of drive vehicles are evolutionary systems. Evolutionary system means the parameters of the shaft are changing in time. The shafts are long and slender. For this reason, they are affected by torsional stress and also by lateral and transversal vibrations [2].

Due to the continuous operations, the shafts have to operate in subcritical speed. Results of previous works, which were also compared to experiments, showed that the propeller shafts represent strong evolutionary systems (increasing the angular velocity of rotation significantly reduces the spectrum of natural frequency relative lateral vibrations) and in practical calculations it is necessary to respect this influence. For that reason, it is not possible to model the shafts using procedures that are commonly reported in the literature, but it is necessary to formulate a model that allows for this effect to be respected. Due to results of previous works and experiments, it is not possible to model the shafts using procedures that are commonly reported in the literature [3], [4], but it is necessary to formulate a model that allows respecting that with increasing the angular velocity of rotation the spectrum of natural frequency relative lateral vibrations is significantly reduced.

Propeller shafts are in a steady state stressed by excitation bending moment's harmonics and their vectors are perpendicular to the rotating plane of a relevant fork Hooks joints. The drive torque mentioned in a steady state is generated due to the transmission flow through Hooks joints and causes lateral oscillations of the propeller shaft in its rotating space. In formulating a mathematical model, it is necessary to start from the assumption of existence of the relative spatial bending vibration in the shaft system $0(\mathrm{x}, \mathrm{y}, \mathrm{z})$, which rotates at an angular speed . If one neglects the Coriolis force and gyroscopic moments acting on the element of the shaft, one can solve the problem in the rotating plane $0(\mathrm{x}, \mathrm{y})$. The instantaneous state of the element is determined by the velocity and the angular velocities. This article aims to build a mathematical model of a coupling shaft to calculate spectral and modal properties of the connecting shaft with respect to the field of centrifugal forces that is causing the addition of natural frequencies of bending vibrations relative to the angular velocity of the shafts rotation.

\section{Formulation of the problem}

Propeller shafts are in a steady state stressed by excitation bending moments harmonic, and their vectors are perpendicular to the rotating plane of a relevant fork Hook's joints (Figure 1).

A model was built on an assumption of existence of the relative spatial bending vibration in the shaft system , (Figure 2), which rotates at an angular speed $\vec{\varphi}_{x}$. The dimensionality of the problem can be reduced from $3 \mathrm{D}$ to $2 \mathrm{D}$ by neglecting the Coriolis force and gyroscopic moments acting on the element of the shaft. Then, one can solve the problem in the rotating plane. The instantaneous state of the element is determined by the angular velocity $\overrightarrow{\dot{\varphi}_{x}}$, the velocity $\overrightarrow{\vec{v}_{x}}$ and the angular velocity $\overrightarrow{\dot{\varphi}_{z s}}$. A mathematical model of a coupling shaft was built in order to calculate the spectral and modal properties of the connecting shaft, including the natural frequency of bending oscillations.

\section{Physical discretization}

The drive shaft, shown in Figure 1 (consider solid bearings), is replaced by a discrete mechanical system with only one degree of freedom. This system is divided into two equal halves, which represent an intangible spring (Figure 3) having the rigidity $\frac{k}{2}$.

\footnotetext{
* ${ }^{2}$ Petr Hruby, ${ }^{2}$ Tomas Nahlik, ${ }^{2}$ Dana Smetanova

${ }^{1}$ Department of Mechanical Engineering, The Institute of Technology and Business, Ceske Budejovice, Czech Republic

${ }^{2}$ Department of Informatics and Natural Sciences, The Institute of Technology and Business, Ceske Budejovice, Czech Republic

E-mail: smetanova@vstecb.cz
} 


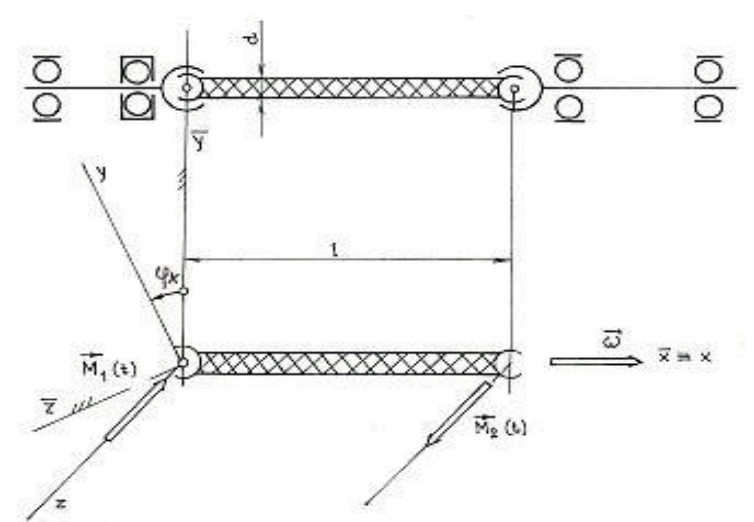

Figure 1 Model of propeller shafts

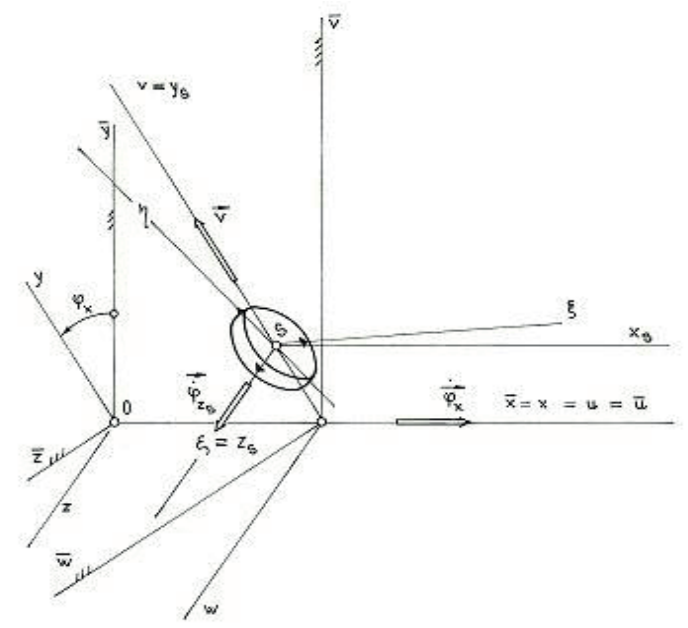

Figure 2 Coordinate system of the shaft

The mass is concentrated to the endpoints of the springs. This means that the two fixed points belongs to the support and two others fixed points will merge into one in the middle of the shaft. This middle point has the mass of $m=\frac{S l_{p}}{2}$, where $S$ is the crosssection area, $l$ is length of the shaft and $\rho$ is density. This model can be simply transformed to model of the spring (Figure 4).

In this case, one can determine the stiffness of this spring as $k=\frac{48 E J}{l^{3}}$, where $E$ is a modulus of elasticity in tension, $J$ is defined as $J=\frac{\pi}{4} r^{4}$ and $l$ is length of the shaft. Assuming the constant angular velocity $\omega$ it is necessary to introduce the moment $\vec{M}$. Now one can write equations for kinetic and potential energy of the spring, respectively, as:

$E_{k}=\frac{1}{2} m \dot{y}^{2}+\frac{1}{2} m\left(y \dot{\varphi}_{x}\right)^{2} \quad E_{p}=\frac{1}{2} k y^{2}$

In addition, equations of motion are written by formulas:

$m \ddot{y}+\left(k-m \omega^{2}\right) y=0, M-2 m y \dot{y} \omega=0$

Equation of relative oscillating movement in rotating plane can be rewritten in the form:

$\ddot{y}+\Omega^{2} y=0$

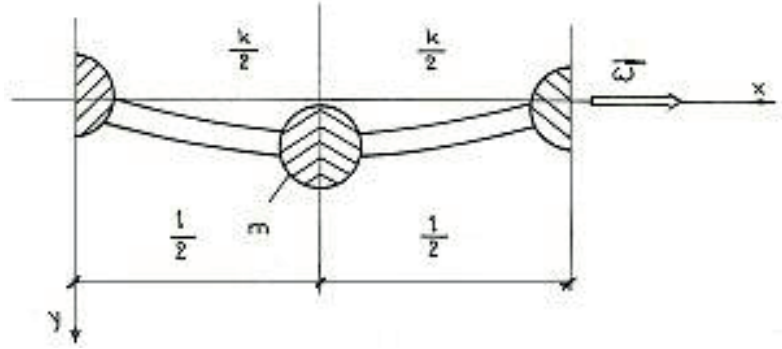

Figure 3 Replacing of the drive shaft by divided system of one degree of freedom

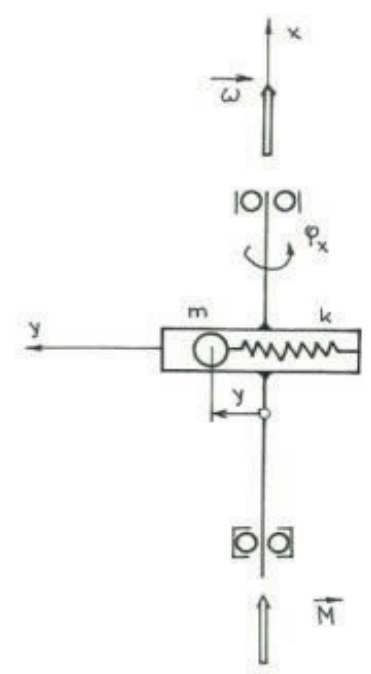

Figure 4 Modelling of the shaft by the spring

where $\Omega=\sqrt{\frac{k}{m}-\omega}$ is the natural frequency of relative undamped oscillations. By modification of this equation one obtains:

$\Omega^{2}+\omega^{2}=\frac{k}{m}$

which is equation of the circle with origin at $O(\omega, \Omega)$ and radius $\frac{k}{m}$.

\section{The test model}

The test model parameters are chosen of the prototype car - Skoda 781. This choice comes from the cooperation with the industry. Parameter of this test model are: $r=0.0105 \mathrm{~m}, l=0.65 \mathrm{~m}, E=2.1 \cdot 10^{11} \mathrm{~Pa}$ and $\rho=7.8 \cdot 10^{3} \mathrm{~kg} \cdot \mathrm{m}^{-3}$. Using these parameters the following was obtained: $J=9 \cdot 10^{-4} \mathrm{~m}^{4}$, $S=3.46 \cdot 10^{-4} \mathrm{~m}^{2}, k=3.3 \cdot 10^{5} \mathrm{Nm}^{-1}, \quad m=0.88 \mathrm{~kg}$ and $\Omega(0)=591.9483 \mathrm{rad} \cdot s^{-1}$ (see Figure 5).

It is also possible to obtain an analytical solution by solving the following equation derived in [5], which is describing the model in Figure 6:

$$
\begin{aligned}
& \frac{\partial^{4} y}{\partial x^{4}}-\frac{\rho S r^{2}}{4 E J} \cdot \frac{\partial^{4} y}{\partial x^{2} \partial t^{2}}-\frac{\rho S r^{2} \omega^{2}}{4 E J} \cdot \frac{\partial^{2} y}{\partial x^{2}}+\frac{\rho S}{E J} \\
& \cdot \frac{\partial^{2} y}{\partial t^{2}}-\frac{\rho S \omega^{2}}{E J} \cdot y=0
\end{aligned}
$$




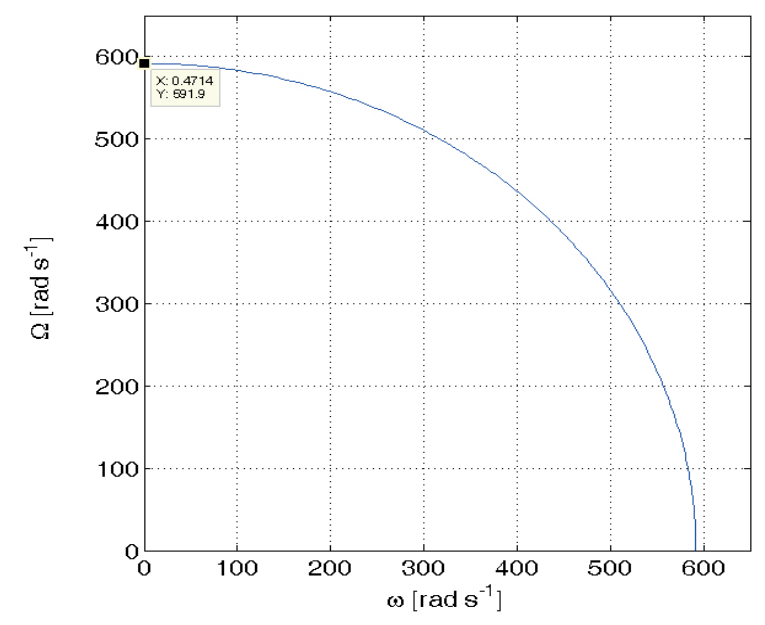

Figure 5 The natural frequency dependence of discrete model's relative transverse vibrations (shown in Figure 4) on the angular velocity of rotation

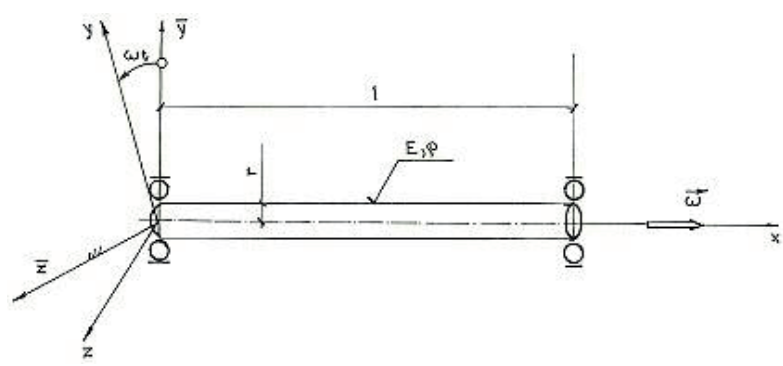

Figure 6 Test model for calculation of propeller shaft's speed resonance

Solution of Equation (5) provides the relation between $\Omega$ and $\omega$ in form $\frac{\Omega^{2}}{R^{2}}+\frac{\omega^{2}}{R^{2}}=1$. For the test model, the final analytic solution is: $R=\frac{r}{2} \sqrt{\frac{E}{\rho}}\left(\frac{\pi}{l}\right)^{2}$.

If one calculates $R$ with given testing parameters, the value $R=636.1432 \mathrm{rad} \cdot \mathrm{s}^{-1}$ is obtained and, by parametrizing with $\omega$, the graph in Figure 7.

\section{The Finite element method}

An element of the shaft in the shape of prismatic section with the circular cross-section is considered (Figure 8).

In this case, the deflection can be described as $y(x, t)=\sum_{i=1}^{4} q_{i}(t) \Phi_{i}(x)$, where $\Phi_{i}(x)$ are the 3 rd order polynomials. For more details see [6]. One can take this model and join it multiple times together to create graduated shaft (Figure 9). The graduated shaft means that the shaft is divided into several parts, which are mathematically described separately. These descriptions of part are linked in the model through the boundary conditions of the parts.

Using the finite element method [7], the whole shaft is then described by a matrix and each part as a sub-matrix. For more details see [6], [8], [9], [10].

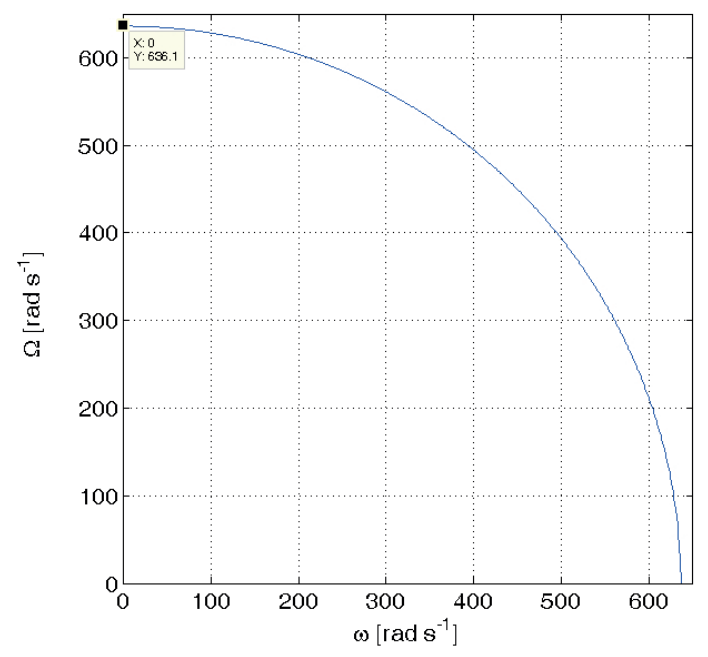

Figure 7 The graph of functional dependence of the angular frequency $(\Omega)$ lateral vibrations in terms of the angular speed of rotation $(\omega)$ of the propeller shaft's test model

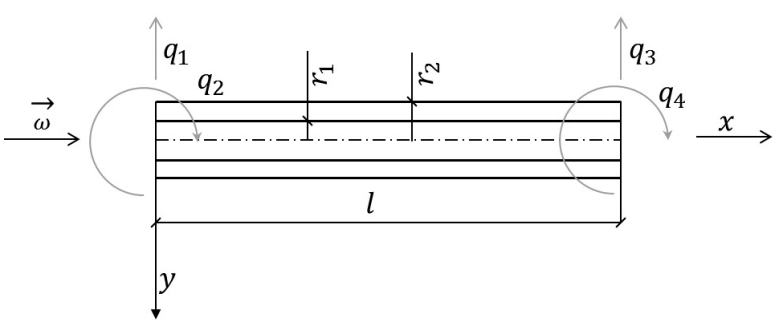

Figure 8 The element of the propeller shaft in the state of combined bending-gyratory vibrations

\section{The Transfer-matrix method}

For calculation using the transfer-matrix method, the model of the shaft is treated as one dimensional continuum [11] of constant circular cross section. It is defined by:

- Geometrical parameters $l[m]$ - the length of the one dimensional continuum $r[\mathrm{~m}]$ - radius of cross section of the shaft.

- Material constants $\rho\left[\mathrm{mkg}^{-3}\right]$ - the material density $E[P a]$ - the modulus of elasticity in tension or compression.

- Operating parameter $\omega\left[\mathrm{rads}^{-1}\right]$ - the angular velocity of rotation of the plane $O(x, y)$ around the axis x.

The solution is sought in the form of

$y(x, t)=Y(x) e^{i \Omega t}$

The solution of Equation (6) can be arranged to the vector of state $-\mathbf{V}(x)$. This vector is bound by the initial vector state to a coordinate of $x=0$ by the relationship:

$\mathbf{V}(x)=\mathbf{H}(x) \mathbf{V}(0)$

and boundary vectors $\mathbf{V}(0), \mathbf{V}(l)$ of the shaft's condition are bound by the transfer matrix of the continuum section $\mathbf{H}(l)$. This means: 

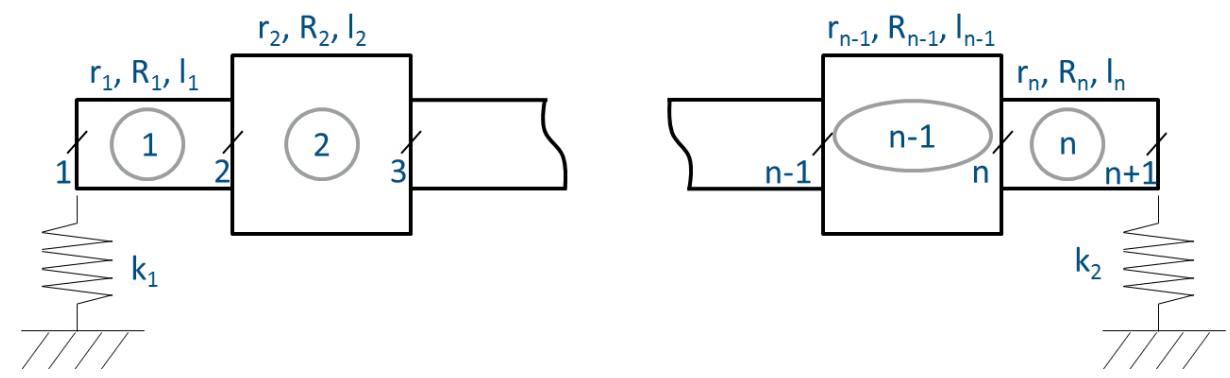

Figure 9 Dynamic model of graduated shaft

$\mathbf{V}(l)=\mathbf{H}(l) \mathbf{V}(0)$

Where

$\mathbf{V}(l)=\left[\begin{array}{c}0 \\ Y^{\prime}(l) \\ 0 \\ -Q(l)\end{array}\right], \mathbf{V}(0)=\left[\begin{array}{c}0 \\ Y^{\prime}(0) \\ 0 \\ -Q(0)\end{array}\right]$

These are boundary vectors for the intended type of the deposit - zero deflection, zero bending moments at the edges. $Y^{\prime}(0), Y^{\prime}(l)$ are the amplitudes of the deflection slope line, $Q(0), Q(l)$ are the amplitudes of the shear forces at the edges of the shaft. From Equation 7, Equation 8 and Equation 9 following statements can be derived:

$\left[\begin{array}{cccc}H_{12} & -H_{14} & 0 & 0 \\ H_{22} & -H_{24} & -1 & 0 \\ H_{32} & -H_{34} & 0 & 0 \\ H_{42} & -H_{44} & 0 & 1\end{array}\right] \cdot\left[\begin{array}{c}Y^{\prime}(0) \\ Q(0) \\ Y^{\prime}(l) \\ Q(l)\end{array}\right]=\mathbf{0}$

The frequency equation can be obtained from the condition of the non-trivial solution.

$$
H_{12} H_{34}-H_{14} H_{32}=0
$$

Based on properties of the shaft and initial condition, the explicit form of the transfer matrices $H_{12}, H_{34}, H_{14}, H_{32}$ can be found. For more details see [12].

\section{Conclusion}

The mathematical model, based on the physical discretization was defined and used for solving the problem of finding the critical speed of rotations. This model was programmed as a script in the GNU Octave. In addition, there is a possibility of using the analytical solution derived from Equation (6). Both of these solutions give the circular dependency of $\Omega$ and $\omega$ (Equation (5) and Equation (7)). The use of Finite element method for modelling the propeller shaft and for calculation of critical speed was attempted. However, this needs a little bit more of programming and calculation. The use Transfer-Matrix method was tried, as well. Using this method should enable modeling of a shaft composed of different parts with different properties. The initial goal is to prepare the scripts for testing. After that, the program should be written, which will be more user friendly than the scripts.

\section{Acknowledgement}

The work presented in this paper was supported by project TA 04010579 of Technology Agency of the Czech Republic and grant IGS201801 of the Institute of Technology and Business in Ceske Budejovice.

\section{References}

[1] EVERNDEN, H. I. F.: The Propeller Shaft or Hooke's Coupling and the Cardan Joint [online]. Proceedings of the Institution of Mechanical Engineers: Automobile Division, 2(1), 100-110, 2006. Available: http://journals.sagepub.com/doi/10.1243/PIME_ AUTO_1948_000_013_02 [accessed 2018-07-09]. https://doi.org/10.1243/PIME_AUTO_1948_000_013_02. ISSN 0367-8822

[2] HADDARA, M. R.: On the Transverse Vibration of a Propeller-Tail Shaft System. Ocean Engineering, 15(2), 119-126, 1988. https://doi.org/10.1016/0029-8018(88)90023-6

[3] HAJEK, E., REIF, P., VALENTA, F.: Flexibility and Strength I / Pruznost a Pevnost I (in Czech). State Publishing House of Technical Literature (SNTL), Prague, 1988.

[4] HAJEK, E., REIF, P., VALENTA, F.: Flexibility and strength II / Pruznost a Pevnost II (in Czech). Czech Technical University in Prague (CVUT), Prague, 1985.

[5] HRUBY, P.: Cardan Coupling Shaft Vibrations in the Rotating Plane. PhD. thesis, University of Mechanical and Electrical Engineering (VSSE), Plzen, 1979.

[6] HRUBY, P., NAHLIK, T., SMETANOVA, D.: Proposal Mathematical Model for Calculation of Modal and Spectral Properties. Post-conference proceedings of extended versions of selected papers of conference Mathematics, Information Technologies and Applied Sciences (MITAV 2017), Czech Republic, 131-140, 2017.

[7] REDDY, J. N.: An Introduction to the Finite Element Method, 2nd ed. McGraw-Hill, New York, 1993. 
[8] HRUBY, P., HLAVAC, Z., ZIDKOVA, P.: Application of the Finite Element Method in Determination of Modal and Spectral Properties of Propeller Shafts Bending Vibrations. Proceedings of the 5th biannual CER Comparative European Research Conference - international scientific conference for Ph.D. students of EU countries, United Kingdom, 132-135, 2016.

[9] HOSCHL, C.: The Use of Small Computers in the Dynamics of Systems. DT CSVTS Prague, 1983.

[10] HRUBY, P.: Bending-Gyratory Vibrations of Shafts in Drives with Joints. University of Mechanical and Electrical Engineering (VSSE), Plzen, 1981.

[11] ZIDKOVA, P., HRUBY, P.: Mathematical Model of One-Dimensional Continuum in State of Combined Bending-Gyratory Vibration / Matematicky Model Jednorozmerneho Kontinua ve Stavu Kombinovaneho Ohybove-Krouziveho Kmitani (in Czech). Proceedings of International Masaryk Conference for Ph.D. Students and Young Researchers (MMK 2016), Czech Republic, 1804$1813,2016$.

[12] HRUBY, P., HLAVAC, Z., ZIDKOVA, P.: The Transfer-Matrix Method in the Application for an One-Dimensional Linear Continuum Speed Resonance. Proceedings of the 5th biannual CER Comparative European Research Conference - international scientific conference for Ph.D. students of EU countries, United Kingdom, 141-145, 2016. 